\title{
Long-wave fluctuations of the two-dimensional Heisenberg antiferromagnet
}

\author{
Victor Belinicher*, Joao da Providencia \\ University of Coimbra, 3000 Coimbra, Portugal
}

\begin{abstract}
We have reduced the Heisenberg model for the quantum antiferromagnet in the leading approximation over $1 / s$ to the spin-rotator model which represents some sort of quantum lattice rotator model. We perform the long-wavelength reduction of this spin-rotator model. An intermediate scale $\Lambda$ was introduced and the short-wavelength antiferromagnetic spin fluctuations were integrated out. As a result, we obtained the continuum nonlinear sigma model with some additional contributions. We presented arguments showing that this model is renormalizable in the framework of $1 / N$ perturbation theory. We separated out all ultraviolet divergencies and expressed all parameters of the theory in terms of the parameters of the primary Heisenberg model, in the one-loop approximation. (C) 2000 Published by Elsevier Science B.V. All rights reserved.
\end{abstract}

Keywords: Antiferromagnet; Disorder; Fluctuations

\section{Introduction}

Paper contains the following basic results:

(1) We have reduced the Heisenberg model for the quantum antiferromagnet on a lattice to some sort of the quantum lattice rotator model (QLRM) [1,2] in the leading approximation over $1 / s$. We shall call this model the spin-rotator model.

(2) We have performed the separation of fluctuations for the spin-rotator model with the help of the introduction of some separation scale $\Lambda$ in momentum space. As a result, we obtain the long-wave nonlinear sigma model with additional contributions from short-wave fluctuations.

(3) We presented arguments showing that the $1 / N$ perturbation theory for the continuous nonlinear sigma model, in the approach based on the introduction of a Lagrange multiplier, for $1+2$ time and space dimensions, in fact contains only logarithmic divergences and is a renormalizable theory.

\footnotetext{
* Corresponding author: Institute of Semiconductor Physics, 630090 Novosibirsk, Russia.

E-mail address: belin@isp.nsc.ru (V. Belinicher)
}

(4) We have performed the separation of divergences in the one-loop approximation and checked the cancellation of the dependence on the cut-off momentum $\Lambda$ due to the contribution of short-wave fluctuations.

(5) We believe that the present approach can help to solve the important problem of the modern theory of antiferromagnetic fluctuations: connection between the well-established microscopic Hamiltonian and quantitative properties of long-wave antiferromagnetic fluctuations.

\section{Derivation of the spin-rotator model}

We consider the spin system which is described by the following Heisenberg Hamiltonian:

$\hat{H}_{\mathrm{Hei}}=J \sum_{\left\langle l l^{\prime}\right\rangle}\left(\hat{\boldsymbol{S}}_{l} \hat{\boldsymbol{S}}_{l^{\prime}}\right), \quad\left(\hat{\boldsymbol{S}}_{l} \hat{\boldsymbol{S}}_{l}\right)=s(s+1)$,

where $\hat{\boldsymbol{S}}_{l}$ are the spin operators, the indices $l, l^{\prime}$ run over the nearest-neighbor sites of a two-dimensional square lattice, $J>0$ is the exchange constant for the antiferromagnetic spin interaction, and $s$ is the magnitude of 
spin. The fluctuation of the quantum antifferomagnet are described by a functional integral over the unit vectors $\boldsymbol{n}_{c, b}, \boldsymbol{n}_{c, b}^{2}=1$ [1] for two antiferromagnetic sublattices $b$ and $c$. Now we can introduce new more convenient variables $\boldsymbol{\Omega}$ and $\boldsymbol{M}$ which realize the stereographic mapping of a sphere:

$\boldsymbol{n}_{b, c}=\frac{ \pm \boldsymbol{\Omega}\left(1-\boldsymbol{M}^{2} / 16 s^{2}\right)+[\boldsymbol{M} \times \boldsymbol{\Omega}] / 2 s}{1+\boldsymbol{M}^{2} / 16 s^{2}}$,

where $\boldsymbol{\Omega}^{2}=1$, and $(\boldsymbol{\Omega M})=0$. In terms of the variables $\boldsymbol{\Omega}$ and $\boldsymbol{M}$ the total Lagrangian $L=L_{\mathrm{kin}}+L_{\text {pot }}$ in the lowest approximation with respect to $\boldsymbol{M}$ has an extremely simple form:

$L_{\boldsymbol{M} \Omega}=\mathrm{i}(\boldsymbol{M} \dot{\boldsymbol{\Omega}})+\frac{1}{2}(\boldsymbol{\Omega} \hat{P} \boldsymbol{\Omega})+\frac{1}{8 s^{2}}(\boldsymbol{M} \hat{Q} \boldsymbol{M})+\cdots$,

$(\hat{Q}, \hat{P})=2 J S^{2} z(1 \pm \hat{\gamma})$,

where $\gamma_{k}=(1 / 2)\left(\cos \left(k_{x} a\right)+\cos \left(k_{y} a\right)\right)$. We can integrate over the field $\boldsymbol{M}$ with the help of some kind of the Faddev-Popov trick, and obtain the final action for the field $\boldsymbol{\Omega}$ and the Lagrange multiplier $\lambda$ :

$L_{\Omega}=2 s^{2}\left(\dot{\boldsymbol{\Omega}} \hat{Q}^{-1} \dot{\boldsymbol{\Omega}}\right)+\frac{1}{2}(\boldsymbol{\Omega} \hat{\boldsymbol{P}} \boldsymbol{\Omega})+\mathrm{i} \lambda \cdot\left(\boldsymbol{\Omega}^{2}-1\right)$.

One can easily recognize in Eq. (4) the Lagrangian of some kind of the quantum lattice rotator model (QLRM) [2]. However, it is different from the standard model due to the momentum dependence of the kinetic term in Eq. (4). We shall call such kind of models the spin-rotator (SR) ones. The SR model as well QLRM is well defined and does not contain any divergences.

\section{Description of long-wave fluctuations}

The Lagrangian of the long-wave sigma model is the universal one and can easily be obtained as a naive long-wave limit (4):

$L_{c}=\frac{\chi_{\perp}}{2}\left(\dot{\boldsymbol{n}}^{2}+c_{\mathrm{s}}^{2}\left(\partial_{i} \boldsymbol{n}\right)^{2}+\mu^{2} \boldsymbol{n}^{2}\right)+\mathrm{i} \lambda\left(\boldsymbol{n}^{2}-1\right)$

where $\partial_{i}$ is the space derivative for $i:=x, y ; c_{\mathrm{s}}$ is the velocity of sound, $\chi_{\perp}$ is the transverse susceptibility, $\mu$ is the inverse correlation length of the $n$-field in a disordered phase [3]. Because the characteristic low-energy scale $E_{\text {low }}=\max (T, \mu) \quad(T$ is the temperature $)$ is much less than the exchange constant $J$ the long-wave antiferromagnetic fluctuations contain many universal properties.

We will use the scale separation based on some sort of Pauli-Villars transformation. Let us choose $\hat{G}=p^{-2}$ and $\hat{G}_{1}=\left(p^{2}\left(1+p^{2} / \Lambda^{2}\right)\right)^{-1}, \hat{G}_{2}=\left(p^{2}+\Lambda^{2}\right)^{-1}$, where $\hat{G}=\hat{G}_{1}+\hat{G}_{2}$, where $\Lambda \ll J$ is the separation scale.

Using this idea we can accurately separate scales in the lattice theory with the Lagrangian (4) having the Lagrangian (5) as long wave limit. In this way we obtained easily the following expression for $\mu$ :

$\mu=T \exp \left(-2 \pi J S^{2} / T N\right), \quad N=3$.

Relation (6) expresses $\mu$ directly in terms of the parameters of the Heisenberg model. We also got the finite expressions for the renormalization constant of the theory.

\section{References}

[1] E. Manousakis, Rev. Mod. Phys. 63 (1991) 1.

[2] S. Chakravarty, B.I. Halperin, D.R. Nelson, Phys. Rev. B 39 (1989) 2344.

[3] A.M. Polyakov, Gauge Fields and Strings, Harwood, New York, 1987. 